\title{
Code-Aided Channel Tracking and Frequency Offset-Phase Noise Elimination for Multicarrier Systems
}

\author{
Mohamed Marey, Mamoun Guenach, Member, IEEE, and Heidi Steendam, Senior Member, IEEE
}

\begin{abstract}
In this contribution, we propose a novel code-aided channel tracking and carrier frequency offset (CFO) and/or phase noise (PN) estimation algorithm for orthogonal frequency-division multiplexing (OFDM) systems. Starting from the maximum-likelihood (ML) principle, we derive a low complexity estimator based on the expectation-maximization (EM) algorithm. It turns out that the estimator receives soft information from the channel decoder to refine the CFO, PN, and channel estimates. Simulation results revealed that the proposed algorithm outperforms the conventional techniques in terms of bit-error rate (BER).
\end{abstract}

Index Terms-Carrier frequency offset, channel tracking, coding, expectation-maximization (EM) algorithm, orthogonal frequency-division multiplexing (OFDM), phase noise.

\section{INTRODUCTION}

$\mathbf{T}$ HE main weakness of orthogonal frequency-division multiplexing (OFDM) is its sensitivity to a carrier frequency offset (CFO) and phase noise (PN) caused by oscillator instabilities. Both impairments lead to a reduction and rotation of the signal amplitude and to a loss of subcarrier orthogonality. This loss introduces intercarrier interference (ICI) which results in a degradation of the global system performance. A frequency offset and/or a phase noise bandwidth as small as a few percent of the subcarrier spacing are sufficient to impair the performance of an OFDM receiver, as pointed out in [1]. In order to operate correctly, an OFDM receiver needs accurate compensation of the offsets in the input signal. Further, a coherent receiver has to track time-varying fading channels in order to accommodate reliable data detection.

Depending on the characteristics of the transmitted signal (pilot-based or not), synchronization and channel estimation approaches can be mainly divided into two categories: data-aided, e.g., [2] and [3], and non-data-aided (blind), e.g., [4] and [5].

\footnotetext{
Manuscript received October 13, 2007; revised June 19, 2008. This work was supported by the European Commission in the framework of the FP7 Network of Excellence in Wireless Communications NEWCOM++ (contract no. 216715), and the work of M. Marey was supported by the Arab Republic of Egypt. The associate editor coordinating the review of this manuscript and approving it for publication was Dr. Yuan-Pei Lin.

M. Marey and H. Steendam are with the Department of Telecommunication and Information Processing, Ghent University, B-9000 Gent, Belgium (e-mail: mohamed@telin.ugent.be; hs@telin.ugent.be).

M. Guenach is with Alcatel-Lucent Bell Labs, Antwerp, Belgium (e-mail: guenach@ieee.org).

Color versions of one or more of the figures in this paper are available online at http://ieeexplore.ieee.org.

Digital Object Identifier 10.1109/LSP.2008.2004517
}

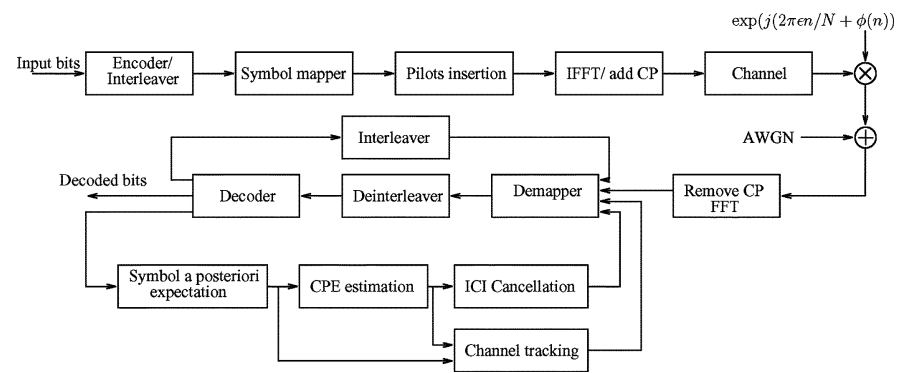

Fig. 1. Conceptual block diagram of the proposed system.

With the advent of powerful error-correcting codes, conventional estimation algorithms cannot always be applied as they may require a significant overhead in terms of the number of pilot symbols if data-aided schemes are used or may require a large amount of received symbols if blind schemes are used. It is thus of interest to develop synchronization and channel estimation schemes by taking into account the presence of the error-correcting codes.

In this letter, we propose a new code-aided channel tracking and $\mathrm{CFO}^{1}$ and/or PN estimation algorithm based on the EM algorithm [6]. The proposed algorithm exploits the soft information provided by the channel decoder to improve the estimation accuracy in an iterative way.

This letter is organized as follows: the system model is described in Section II. The estimation algorithm is derived in Section III. Simulation results are provided in Section IV. Finally, we end with conclusions in Section V.

\section{SySTEM MODEL}

Fig. 1 depicts the discrete time base-band equivalent block diagram for the proposed OFDM system. At the transmitter, a binary information sequence $\mathbf{b}=\left[b(0), b(1), \cdots, b\left(N_{b}-1\right)\right]^{T}$ is passed through a channel encoder with coding rate $R_{c}$. The resulting binary codeword $\mathbf{c}=\left[c(0), c(1), \cdots, c\left(N_{c}-1\right)\right]^{T}$, $N_{c}=N_{b} / R_{c}$ is then interleaved and mapped onto $N_{d}$ symbols $\mathbf{d}=\left[d(0), d(1), \cdots, d\left(N_{d}-1\right)\right]^{T}$, where $d(i)$ belongs to a $2^{q}$-point constellation $\xi, N_{d}=N_{c} / q$. We further assume that a total of $N_{p}$ pilot symbols $\mathbf{p}=\left\{p(0), p(1), \cdots, p\left(N_{p}-1\right)\right\}$ are inserted at known locations in each OFDM symbol. The set

\footnotetext{
${ }^{1}$ The $\mathrm{CFO}$ can be split into an integer part and a fractional part with respect to the OFDM subcarrier spacing. This work is focused on the fractional CFO only.
} 
of subcarrier indices on which pilots are transmitted is denoted as $\Gamma$. The resulting block $\mathbf{a}=[a(0), a(1), \cdots, a(N-1)]^{T}$ of $N=N_{d}+N_{p}$ symbols is then transformed by an $N$-point inverse fast Fourier transform (IFFT), yielding

$$
\mathbf{s}=\sqrt{\frac{1}{N+\nu}} \mathbf{F a}
$$

where $a\left(n_{k}\right)=d(k), k=0, \ldots, N_{d}-1$, and $n_{k} \notin \Gamma$, $a\left(n_{k}\right)=p(k), k=0, \ldots, N_{p}$, and $n_{k} \in \Gamma$, and $\mathbf{F}$ is an $N \times N$ matrix with $F_{m, n}=e^{j 2 \pi m n / N}$. The OFDM symbol $\mathbf{s}$ is extended using a cyclic prefix of $\nu$ samples, such that $s(n)=s(n+N) \forall n=-\nu, \cdots,-1$. The transmitted OFDM symbol $\mathbf{s}=[s(-\nu), \ldots, s(N-1)]^{T}$ propagates through an $L$-tap channel with overall channel impulse response (CIR) $\mathbf{h}=[h(0), \ldots, h(L-1)]^{T}$. The time-domain received vector $\mathbf{r}$ can be written as

$$
\mathbf{r}=\operatorname{diag}\left(\left\{e^{j(2 \pi \epsilon n / N+\phi(n))}\right\}_{n=-\nu}^{N+L-2}\right)[\mathbf{s} \star \mathbf{h}]+\mathbf{w}
$$

where $\star$ is the convolution operation, $\phi(n)$ is the phase noise, $\epsilon$ is the CFO normalized to the OFDM subcarrier spacing, and $\mathbf{w}$ is the additive white Gaussian noise (AWGN) contribution. After removing the cyclic prefix and applying FFT, the demodulated vector $\mathbf{R}$ can be written as

$$
\mathbf{R}=\sqrt{\frac{N}{N+\nu}} I(0) \mathbf{A} \mathbf{\Omega} \mathbf{h}+\mathbf{V}+\mathbf{W}
$$

where $\mathbf{A}=\operatorname{diag}(\mathbf{a}), \boldsymbol{\Omega}$ is an $N \times L$ matrix with $\Omega_{k, l}=$ $e^{-j 2 \pi k l / N}, \mathbf{V}$ is the ICI, and $\mathbf{W}$ is the AWGN contribution. The ICI contribution at subcarrier $k^{\prime}$ can be written as

$$
V\left(k^{\prime}\right)=\sum_{k=0, k \neq k^{\prime}}^{N-1} a(k) H(k) I\left(k-k^{\prime}\right)
$$

where $H(k)$ is the frequency domain CIR at subcarrier $k$ and

$$
I(i)=\frac{1}{\sqrt{N(N+\nu)}} \sum_{n=0}^{N-1} e^{j(2 \pi(i+\epsilon) n / N+\phi(n))} .
$$

Equation (3) shows that the presence of CFO and/or PN affects the received useful signal. Both impairments rotate, attenuate the useful signal, and add extra noise referred as ICI.

\section{CHANNEL TRACKING AND CFO-PN ELIMINATION}

In this section, we give a brief outline of the EM algorithm and apply it to the observation vector $\mathbf{R}=[R(0), \cdots, R(N-$ 1) $]^{T}$ from (3) to estimate the common phase error $I(0)$ and track the CIR. Note that strictly speaking, the entire sequence $I(i)$, $i=0, \ldots, N-1$ needs to be estimated. However, we will show that there is no easy closed-form solution to the joint estimation of such sequence together with the channel. Therefore, we will resort to a low complex solution of the EM by focusing on the estimation of the CPE $I(0)$ and the CIR tracking.

\section{A. EM Algorithm: Principle}

The EM algorithm is an iterative approach to acquire the maximum-likelihood (ML) estimate of a parameter $\lambda$ from an observation $\mathbf{r}$ [6]. It is based on the concept of the so-called missing (or unobserved data) a, such that, if the missing data were known, estimating $\lambda$ would be easy. However, since we do not know the missing data, an iterative approach starting from an initial estimate of $\boldsymbol{\lambda}$ (say, $\widehat{\boldsymbol{\lambda}}_{(1)}$ ) is used. Consider $\mathbf{r}$ as the "incomplete" observation and $\mathbf{z} \doteq[\mathbf{r}, \mathbf{a}]$ as the "complete" observation. At iteration $\iota$, the EM algorithm consists of two steps:

1) E-step: given the current estimate $\widehat{\lambda}_{(\iota)}$ and "incomplete" observation $\mathbf{r}$, we first take the expectation of the $\log$-likelihood of the complete data $\mathbf{z} \doteq[\mathbf{r}, \mathbf{a}]$ with respect to the unknown data $\mathbf{a}$ :

$$
Q\left(\lambda \mid \widehat{\boldsymbol{\lambda}}_{(\iota)}\right)=\mathrm{E}_{\mathbf{a}}\left[\log \mathrm{P}(\mathbf{z} \mid \boldsymbol{\lambda}) \mid \mathbf{r}, \widehat{\boldsymbol{\lambda}}_{(\iota)}\right]
$$

2) M-step: we maximize $Q\left(\boldsymbol{\lambda} \mid \widehat{\boldsymbol{\lambda}}_{(\iota)}\right)$ with respect to $\lambda$ to find a new estimate:

$$
\widehat{\boldsymbol{\lambda}}_{(\iota+1)}=\arg \max _{\boldsymbol{\lambda}}\left\{Q\left(\boldsymbol{\lambda} \mid \widehat{\boldsymbol{\lambda}}_{(\iota)}\right)\right\} \text {. }
$$

The EM algorithm iterates until the estimate has converged or a certain stopping criterion has been met.

\section{B. Soft Information-Aided Algorithm}

In the problem under consideration, the parameters to be estimated are $\mathbf{h}$ and the set $\mathbf{I}$ defined as $\mathbf{I}=\{I(i), i=0, \ldots, N-$ $1\}$. Based on the observation model (3), we write

$$
\log \mathrm{P}(\mathbf{R}, \mathbf{a} \mid \mathbf{I}, \mathbf{h}) \propto \log \mathrm{P}(\mathbf{R} \mid \mathbf{a}, \mathbf{I}, \mathbf{h})
$$

and

$$
\log \mathrm{P}(\mathbf{R} \mid \mathbf{a}, \mathbf{I}, \mathbf{h}) \propto-\left\|\mathbf{R}-\mathbf{V}-\sqrt{\frac{N}{N+\nu}} I(0) \mathbf{A} \Omega \mathbf{h}\right\|^{2} .
$$

Plugging (8) and (9) in (6) yields

$$
\begin{aligned}
Q\left(\mathbf{I}, \mathbf{h} \mid \widehat{\mathbf{I}}_{(\iota-1)}, \widehat{\mathbf{h}}_{(\iota-1)}\right)= & -\|\mathbf{R}\|^{2}-\| \widetilde{\mathbf{V} \|^{2}}+2 \Re\left\{\mathbf{R}^{H} \widetilde{\mathbf{V}}\right\} \\
& -\frac{N}{N+\nu}|I(0)|^{2} \mathbf{h}^{H} \boldsymbol{\Omega}^{H} \widetilde{\mathbf{A}^{H}} \mathbf{A} \boldsymbol{\Omega} \mathbf{h} \\
& +2 \Re\left\{\sqrt{\frac{N}{N+\nu}} \mathbf{R}^{H} I(0) \widetilde{\mathbf{A}} \boldsymbol{\Omega} \mathbf{h}\right\} \\
& -2 \Re\left\{\sqrt{\frac{N}{N+\nu}} I(0) \widetilde{\mathbf{V}^{H}} \mathbf{A} \boldsymbol{\Omega} \mathbf{h}\right\}
\end{aligned}
$$

where $\Re\{x\}$ is the real value of the complex number $x$, and the a posteriori expectation of a matrix $\mathbf{M}$, denoted $\widetilde{\mathbf{M}}$, with entries that depend on the data $\mathbf{a}$ is defined as

$$
\widetilde{\mathbf{M}}=\int \mathbf{M P}\left(\mathbf{a} \mid \mathbf{R}, \widehat{\mathbf{I}}_{(\iota-1)}, \widehat{\mathbf{h}}_{(\iota-1)}\right) d \mathbf{a} .
$$

Based on (10), we update estimates of $\mathbf{I}$ and $\mathbf{h}$

$$
\left[\widehat{\mathbf{I}}_{(\iota)} \widehat{\mathbf{h}}_{(\iota)}\right]=\arg \max _{\mathbf{h}, \mathbf{I}}\left\{Q\left(\mathbf{I}, \mathbf{h} \mid \widehat{\mathbf{I}}_{(\iota-1)}, \widehat{\mathbf{h}}_{(\iota-1)}\right)\right\} .
$$


As can be observed in (12) and (10), there is no closed-form solution for the estimation of $\mathbf{I}$ and $\mathbf{h}$ because of the higher order components $I(i)$ with $1 \leq i \leq N-1$. To overcome the complexity, we restrict the estimation to the channel and the $\mathrm{CPE}^{2} I(0)$ and propose the following approximations in (10): $\|\widetilde{\mathbf{V}}\|^{2} \approx\left\|\widehat{\mathbf{V}}_{(\iota-1)}\right\|^{2}, \widetilde{\mathbf{V}} \approx \widehat{\mathbf{V}}_{(\iota-1)}$, and $\widetilde{\mathbf{V}^{H}} \mathbf{A} \approx \widehat{\mathbf{V}}_{(\iota-1)}^{H} \widetilde{\mathbf{A}}$, where $\widehat{\mathbf{V}}_{(\iota-1)}$ can be interpreted as the estimated ICI based on the available APPs $\mathrm{P}\left(\mathbf{a} \mid \mathbf{R}, \widehat{\mathbf{I}}_{(\iota-1)}, \widehat{\mathbf{h}}_{(\iota-1)}\right)$ and the previous estimates $\widehat{\mathbf{I}}_{(\iota-1)}$ and $\widehat{\mathbf{h}}_{(\iota-1)}$. Using $\widehat{\mathbf{h}}_{(\iota-1)}$ instead of $\mathbf{h}$ in $(10)$, a simple sub-optimum closed-form expression of $I(0)$ is given by

$$
\begin{aligned}
\widehat{I(0)}{ }_{(\iota)}=\sqrt{\frac{N+\nu}{N}} & \left(\widehat{\mathbf{h}}_{(\iota-1)}^{H} \boldsymbol{\Omega}^{H} \widetilde{\left.\mathbf{A}^{H} \mathbf{A} \boldsymbol{\Omega} \widehat{\mathbf{h}}_{(\iota-1)}\right)^{-1}}\right. \\
& \times \widehat{\mathbf{h}}_{(\iota-1)}^{H} \mathbf{\Omega}^{H} \widetilde{\mathbf{A}^{H}}\left(\mathbf{R}-\widehat{\mathbf{V}}_{(\iota-1)}\right) .
\end{aligned}
$$

The updated channel estimate can easily be determined by setting the differentiation of (10) with respect $\mathbf{h}$ to zero and using $\widehat{I(0)}_{(\iota)}$ as an estimate of $I(0)$

$$
\begin{aligned}
\widehat{\mathbf{h}}_{(\iota)}=\sqrt{\frac{N+\nu}{N}}\left(\widehat{I(0)}{ }_{(\iota)} \boldsymbol{\Omega}^{H} \widetilde{\mathbf{A}^{H}} \mathbf{A} \boldsymbol{\Omega}\right)^{-1} \boldsymbol{\Omega}^{H} \widetilde{\mathbf{A}^{H}} \\
\times\left(\mathbf{R}-\widehat{\mathbf{V}}_{(\iota-1)}\right) .
\end{aligned}
$$

Note that, in practice, the matrices $\widetilde{\mathbf{A}}$ and $\widetilde{\mathbf{A}^{H}} \mathbf{A}$ are obtained by replacing each entry of the main diagonal for both $\mathbf{A}$ and $\mathbf{A}^{H} \mathbf{A}$ with the corresponding a posteriori expectations $\mu(k)$ and $\psi(k)$, respectively, which are obtained by exploiting the a posteriori probabilities (APPs) computed by the decoder

$$
\begin{aligned}
& \mu(k)=\sum_{\omega \in \xi} \omega \cdot \mathrm{P}\left(a(k)=\omega \mid \mathbf{R}, \widehat{\mathbf{I}}_{(\iota-1)}, \widehat{\mathbf{h}}_{(\iota-1)}\right) \\
& \psi(k)=\sum_{\omega \in \xi}|\omega|^{2} \cdot \mathrm{P}\left(a(k)=\omega \mid \mathbf{R}, \widehat{\mathbf{I}}_{(\iota-1)}, \widehat{\mathbf{h}}_{(\iota-1)}\right) .
\end{aligned}
$$

Initialization: The initial value of $\widehat{I(0)}$ can be estimated from (13) by setting the entries in $\widetilde{\mathbf{A}}$ and $\widehat{\mathbf{A}^{H}} \mathbf{A}$ corresponding to the pilot positions to the value of the pilots, and all other entries to zero. This is referred to as the pilot estimator. In the absence of pilot symbols, i.e., if $\Gamma$ is empty, we assume that the initial value of $\widehat{I(0)}$ equals one: in the first iteration, we decode the received data without having any information about the CFO and/or PN. This is referred to as the blind estimator. Further, for both pilot and blind estimators, we assume that the channel is fixed during an OFDM symbol and slowly changes from OFDM symbol to OFDM symbol. Hence, as an initial estimate for the channel, we use the channel estimate from the previous OFDM symbol. ${ }^{3}$ To update the ICI vector, we consider two scenarios.

${ }^{2}$ From $I(0)$, we try to estimate $\epsilon$. As the phase noise $\phi(n)$ in practical situations is typically small as compared to the CFO, the estimate of $\epsilon$ based on $I(0)$ will typically be acceptable. In many practical applications, the phase noise rate is smaller than $10^{-2}$; the specifications for IEEE 802.11a and DVB-T require phase noise rate values in the range $10^{-4} \ldots 10^{-3}$. In such a case, the effect of the ICI term resulting from the PN diminishes [7]. Neglecting the presence of the phase noise, we can reconstruct the ICI vector based on the estimates of $\epsilon$, $\mathbf{h}$, and the decoder output.

${ }^{3}$ In the first OFDM symbol, we assume that the CIR is estimated using another estimator, e.g., based on an all pilots OFDM symbol.
Absence of $P N$ : In the absence of phase noise, $I(0)$ in (5) can be rewritten as

$$
I(0)=\frac{1}{\sqrt{N(N+\nu)}} \frac{\sin (\pi \epsilon)}{\sin (\pi \epsilon / N)} e^{j \pi \epsilon(N-1) / N} .
$$

Accordingly, at iteration $\iota$ the estimated CFO $\hat{\epsilon}_{(\iota)}$ can be obtained as

$$
\hat{\epsilon}_{(\iota)}=\frac{N}{\pi(N-1)} \arg \left(\widehat{I(0)}_{(\iota)}\right) .
$$

Based on $\hat{\epsilon}_{(\iota)}$ and the decoder output, the ICI term in (4) can be calculated and subtracted from the received signal to improve the estimation accuracy in the next iteration.

Presence of PN: Since, in the presence of PN, we do not have information about the high-frequency components of the PN, the estimate of the ICI vector $\widehat{\mathbf{V}}_{(\iota)}$ cannot be obtained. However, it is possible to estimate the CFO from (18), such that the ICI caused by the CFO can be mitigated as if no PN was present. Note that in this case, the estimate of the CFO will be affected by the PN. If no CFO is present, we neglect the ICI vector in all iterations $\left(\widehat{\mathbf{V}}_{(\iota)}=\mathbf{0}\right)$, as in [7].

\section{Simulation Results}

To validate the proposed algorithm, we have carried out Monte Carlo simulations over frequency-selective channels. We consider an OFDM system, using a convolutional code with constraint length 5 , rate $1 / 2$, and polynomial generators $(23)_{8}$ and $(35)_{8}$. The BCJR algorithm is used for decoding. A block length of $N_{b}=128$ information and pilot bits was chosen, leading to $N_{c}=256$ coded bits. The coded bits are Gray-mapped on a 16-QAM constellation, resulting in $N_{d}+N_{p}=64$ symbols. We take $N_{p}=4$ for the pilot estimator while $N_{p}=0$ for the blind estimator. The channel consists of $L=4$ statistically independent taps, each being a zero-mean complex Gaussian random variable with an exponential power delay profile

$$
\sigma^{2}(l)=E_{h} \exp (-l / 5), \quad l=0, \ldots, L-1
$$

where $E_{h}$ is chosen such that the average energy per subcarrier is normalized to unity. To avoid ISI, a cyclic prefix of length $\nu=L-1$ is employed. The time-selective nature of the channel is modeled through the following first-order Markov model for $m>0[8]:$

$$
h^{(m)}(l)=\alpha h^{(m-1)}(l)+\sqrt{1-\alpha^{2}} \sigma^{2}(l) w_{k}^{(m)}
$$

where $^{4}\left\{w_{k}^{(m)}\right\}_{k=0}^{L-1}$ is a sequence of zero mean complex AWGN samples with $\left[\left|w_{k}^{(m)}\right|^{2}\right]=1$. The parameter $\alpha$ determines the time correlation function of the fading process. For instance, for Jake's model, we have $\alpha=J_{0}\left(2 \pi f_{d}\right)$, where $f_{d}$ is the Doppler shift normalized to the OFDM symbol duration, and $J_{0}($.$) is the$ zeroth-order Bessel function of the first kind.

Fig. 2 depicts the bit-error rate (BER) performance for both estimators with a Wiener phase noise rate $\beta=0.002$, a CFO

\footnotetext{
${ }^{4} m$ denotes to the time index of the OFDM symbol. For notational convenience, we drop $m$ in all equations.

${ }^{5}$ The Wiener PN rate is defined as the Wiener PN bandwidth normalized to the OFDM subcarrier spacing [1], [7].
} 


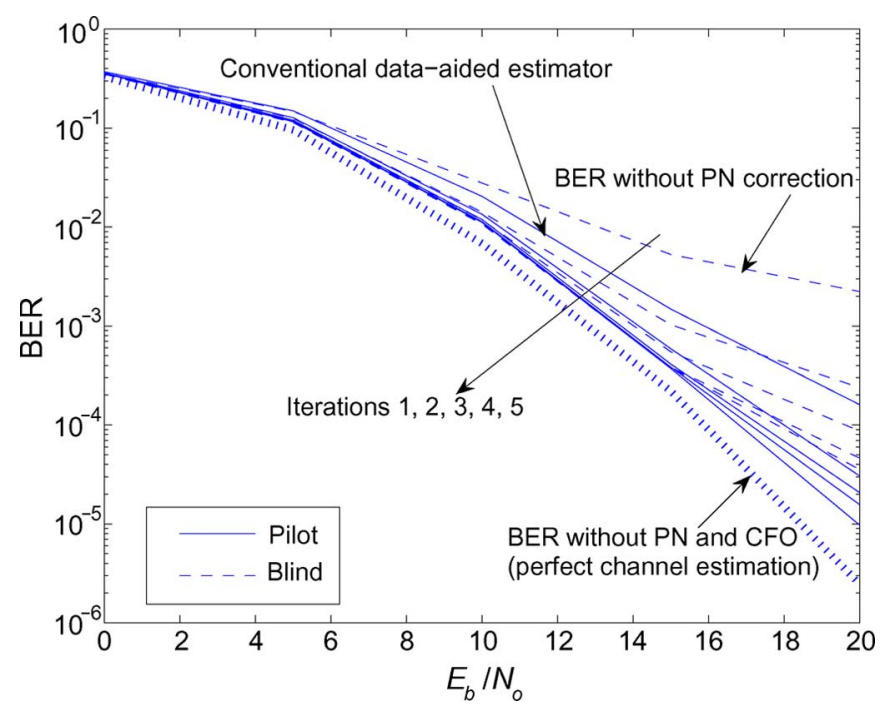

Fig. 2. BER as function of $E_{b} / N_{0}$ with phase noise rate $\beta=0.002, \epsilon=0.05$, and $f_{d}=0.01(\alpha=0.999)$. In the blind estimator, we set the initial value of $\widehat{I(0)}$ equal to one.

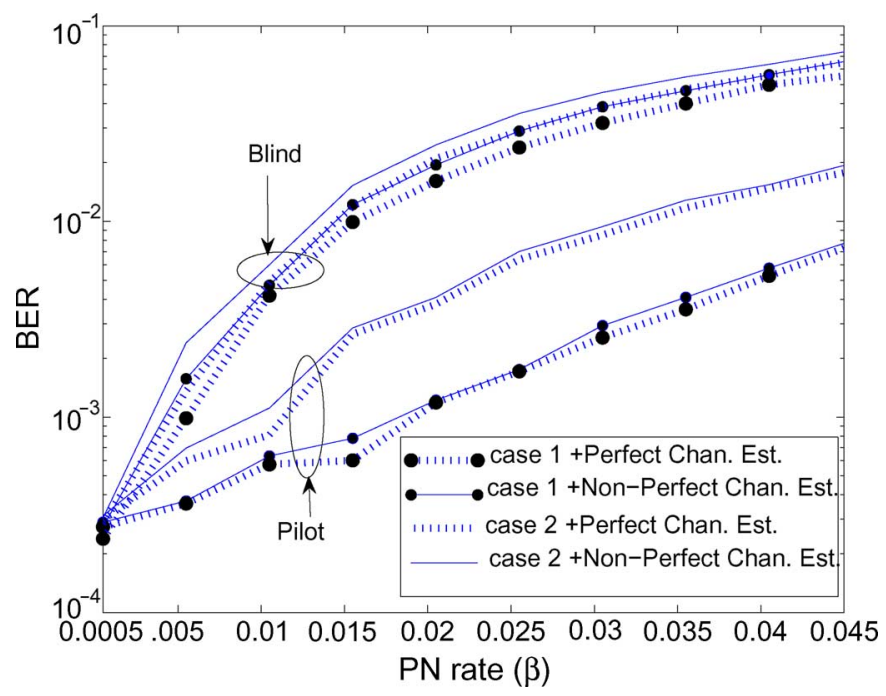

Fig. 3. BER with PN rate at $E_{b} / N_{0}=15 \mathrm{~dB}, \epsilon=0.05$, and $f_{d}=0.01(\alpha=$ $0.999)$ for iteration 5 . In the blind estimator, we set the initial value of $\widehat{I(0)}$ equal to one.

$\epsilon=0.05$, and a normalized Doppler shift $f_{d}=0.01$ as a function of $E_{b} / N_{o}$. We also show the BER that corresponds to perfect channel estimation in the absence of CFO and PN. A strong improvement of the performance for both estimators is achieved after only three iterations. Further, we notice that the pilot estimator outperforms the blind estimator. The EM code-aided pilot estimator is able to reduce the BER degradation to less than $1 \mathrm{~dB}$ after five iterations over a wide range of $E_{b} / N_{o}$.

Fig. 3 shows the BER performance as a function of the phase noise rate $\beta$ at $\epsilon=0.05, f_{d}=0.01$, and $E_{b} / N_{0}=15$ for perfect and nonperfect channel estimation scenarios at iteration 5 . We consider two cases. In the first case, we estimate the CFO, $\epsilon$, from (18) and, based on the decoder output, we perform ICI cancellation as if there is no PN. In the second case, we estimate the CPE, $I(0)$, resulting from both $\mathrm{CFO}$ and $\mathrm{PN}$, and we do not perform ICI cancellation since the high-frequency components of PN are not known. As can be observed from the figure, the BER corresponding to the first case outperforms the second case for both blind and pilot estimators. There is a noticeable difference between cases 1 and 2 for the pilot estimator especially at high PN rate. However, this difference is small for the blind estimator. This occurs because at high PN rate, our approximation for the blind estimator $\left(\widehat{I(0)}_{(1)}=1\right)$ is not good enough to initialize the EM algorithm, such that the CFO component cannot be estimated properly, and the ICI estimate strongly deviates from the true ICI: ICI cancellation is usefulness. Further, we notice that the BER performance for the two cases for both estimators is approximately the same at $\beta=0.0005$. This can be explained as at low PN rate and small CFO, the ICI term is small as compared to the useful signal. Accordingly, performing ICI cancellation does not give a significant improvement on the BER performance.

\section{CONCLuSions}

A novel soft information-aided channel tracking and CFO and/or PN estimation algorithm for OFDM systems is proposed. Based on the proposed low complex EM algorithm, the receiver iterates between data detection and estimation, with the exchange of soft information. The results indicate that the proposed algorithm achieves a significant improvement in BER performance after three iterations as compared to conventional data-aided algorithms. Further, it turns out that it is better to use a small amount of pilots to initialize the proposed low complex EM algorithm rather than setting the initial PN and CFO estimates to zero. The proposed scheme can work with any detector as long as the detector is able to compute the APPs of the data symbols.

\section{REFERENCES}

[1] T. Pollet, M. Van Bladel, and M. Moeneclaey, "BER sensitivity of OFDM systems to carrier frequency offset and Wiener phase noise," IEEE Trans. Commun., vol. 43, no. 2, pp. 191-193, Apr. 1995.

[2] H. Minn, V. Bhargava, and K. Lataief, "A robust timing and frequency synchronization for OFDM systems," IEEE Trans. Wireless Commun., vol. 2, no. 4, pp. 822-838, Jul. 2003.

[3] Y. Li, "Pilot-symbol-aided channel estimation for OFDM in wireless systems," IEEE Trans. Veh. Technol., vol. 49, no. 3, pp. 1207-1215, Jul. 2000.

[4] J. van de Beek, M. Sandell, and P. Borjesson, "ML estimation of time and frequency offset in OFDM systems," IEEE Trans. Signal Process., vol. 45, no. 7, pp. 1800-1805, Jul. 1997.

[5] C. Li and S. Roy, "Subspace-based blind channel estimation for OFDM by exploiting virtual carriers," IEEE Trans. Wireless Commun., vol. 2, no. 1, pp. 141-150, Jan. 2003.

[6] T. Moon, "The expectation maximization algorithm," IEEE Signal Process. Mag., vol. 13, no. 6, pp. 47-60, Nov. 1996.

[7] P. Robertson and S. Kaiser, "Analysis of the effects of phase noise in Orthogonal Frequency Division Multiplexing (OFDM) systems," in Proc. IEEE Int. Conf. Communications, ICC, Seattle, WA, 1995.

[8] H. Wang and P. Chang, "On verifying the first-order Markovian assumption for a Rayleigh fading channel model," IEEE Trans. Veh. Technol., vol. 45, no. 2, pp. 353-357, May 1996. 